\title{
DIGITIZATION OF BLOCKS AND VIRTUAL ANASTYLOSIS OF AN ANTIQUE FACADE IN PONT-SAINTE-MAXENCE (FRANCE)
}

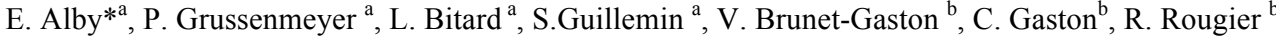 \\ a Photogrammetry and Geomatics Group, ICube Laboratory UMR 7357, INSA Strasbourg, France \\ (emmanuel.alby, pierre.grussenmeyer, laurent.bitard, samuel.guillemin)@insa-strasbourg.fr \\ ${ }^{\mathrm{b}}$ National Institute for Preventive Archaeological Research (INRAP) \\ (veronique.gaston, christophe.gaston, richard.rougier)@inrap.fr
}

\section{COMMISSION II}

KEY WORDS: Close Range Photogrammetry, Laser Scanning, Archaeology, Anastylosis, Simulation

\begin{abstract}
:
This paper is dedicated to the digitization of blocks and virtual anastylosis of an antique façade in Pont-Sainte-Maxence (France). In 2014 during the construction of a shopping center, the National Institute for Preventive Archaeological Research (INRAP) discovered a Gallo-Roman site from the $2^{\text {nd }}$ century AD. The most interesting part of the site for the study is a façade of 70 meters long by nearly 10 meters high. The state of the conservation of the blocks of the façade makes them exceptional due to the question raised by the collapse. Representative and symbolic blocks of this building have been selected for a virtual anastylosis study. The blocks discovered belong to different types: decorated architectural blocks, monumental statuary elements and details of very fine decorations. The digital reproduction of the façade will facilitate the formulation of hypothesis for the collapse of the structure. The Photogrammetry and Geomatics Group from INSA Strasbourg is in charge of the digitization, the anastylosis and the development of exploratory methods for understanding the ruin of the façade. To develop the three-dimensional model of the facade, approximately 70 blocks of various dimensions were chosen by the archaeologists. The choice of the digitization technique is made according to the following pragmatic criterion: the movable objects are acquired with a scan-arm or a hand-held scanner in the laboratory and the largest blocks are recorded by photogrammetry at the repository near Paris. The expected types of deliverables are multiple: very accurate 3D models with the most faithful representation to document the objects in the best way and with optimized size model allowing easy handling during anastylosis tests. The visual aspect of the models is also a very important issue. Indeed, textures from photos are an excellent way to bring about the realism of the virtual model, but fine details of the object are sometimes blurred by the uniformity of the color of the original material. Acquisition by hand-held scanner does not provide the textures (they must be acquired according to a complementary process). The data types are therefore different depending on the acquisition. The type of rendering of the models depends therefore on precise choices to be defined optimally. After the acquisition, hypothesis for the construction of the façade must be validated and / or adapted by the anastylosis of the digitized blocks. Different cases must be taken into account. First, the reconstruction of broken blocks is done by adjusting the recovered fragments. If all the fragments discovered are close to the initial shape of the block, the process is assimilated to a puzzle of complex surfaces. If the fragments have no contact but are an integral part of the block, the proportion of hypotheses in relation to the contact pieces is changed. And finally, if the blocks are to be assembled together by superposition and thanks to a common plan, as assumed during the construction, the restitution could be based on the positions of discoveries and hypotheses based on the architectural knowledge of this period. Each of these three methods of reconstruction involves different processes. The three-dimensional model will be validated by the positioning of the blocks and extended according to the actual dimensions of the façade. Different collapse scenarios will result from this study.
\end{abstract}

\section{INTRODUCTION}

Preventive archeology, through the systematic field survey before construction, is a context that promotes surprising discoveries. The Gallo-Roman site of the 2nd century AD, discovered at Pont-Sainte-Maxence, $50 \mathrm{~km}$ North of Paris, is an unexpected testimony to this period. The most interesting part of the site for the study is a façade of 70 meters long by nearly 10 meters high, composed of 17 open arcades with 2 blind arcades at both extremities. The particularity of the discovery is that the blocks of the facade are very well preserved. The preservation raises questions, particularly with respect to an early ruin, surprising in view of the mastery of the construction by its builders. The quality of the blocks gave the impetus to start a project of digitization and exploitation of the models.

The objective is to understand the causes of the premature fall of the facade. The characteristics of this discovery make it specific. Depending on the dimensions of the elements to be digitized, various acquisition techniques have been adopted. The project deals with the $3 \mathrm{D}$ model rendering aspects as well as the use of the block models to reconstruct the facade. As the project is in progress, a discussion about the falling mechanism design will also be addressed.

\section{PRESENTATION OF THE PROJECT}

The purpose of preventive archaeology is to detect and undertake the scientific study of archaeological remains that might otherwise be destroyed by land development works. Following a decision made by the French State, archaeologists from INRAP intervene on a site in order to safeguard its archaeological heritage. The systematic interventions often deliver unexpected findings. This is what happened during preliminary surveys to the construction of a shopping center in Pont-Sainte-Maxence (France) in 2014. 


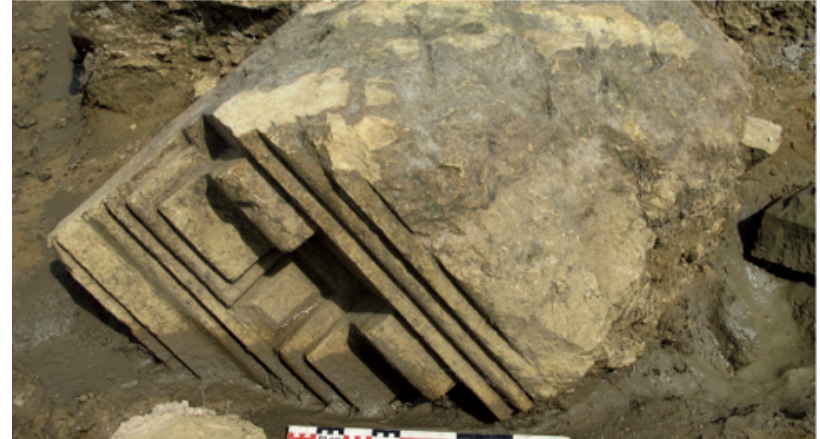

Figure 1. Example of block just after its discovery in the field (Photo: V. Brunet-Gaston, Inrap)

A great number of limestone blocks from a monumental GalloRoman façade were found a few tens of centimeters only below the ground level. The particularity of the discovery is that the blocks of the facade are very well preserved.

This state of conservation can be considered as a first research question. The second question is related to the fact that the facade fell so shortly after its construction. Indeed, the GalloRoman architecture is either in elevation, or ruined or later reused. In both cases, the blocks are usually very eroded. In Pont-Sainte-Maxence, the blocks were almost new at the collapse of the façade and due to the sandy soil of the Oise valley, some pigments and coated were still maintained at the time of discovery. Their layout at the discovery varies according to the types of building blocks. Indeed, for the lower parts, the blocks are missing and have obviously been reused elsewhere. For the higher parts, a large number of blocks have been found in an order respecting the initial superposition. If the logic of the elevation of the facade was readable during the discovery, several other blocks were found in unexpected places. The objective of our project in this context is summarized in the following sentence: the three-dimensional modeling of the blocks must be done to confirm the hypothesis of reconstruction of the facade. The 3D model obtained will be used to support hypothesis for the falling mechanism of the building. The most relevant hypothesis will be verified by the simulation of the fall of the blocks and the confrontation between their supposed trajectory and their arrangement as discovered in 2014

\section{RECORDING}

The number of blocks to be acquired is close to 70 , but this number varies according to the complementary fragments linked with the elements to be digitized. The blocks have been removed a short time after the discovery of the site, and stored by the local authorities in a repository. The acquisition depends on the current access to the stored blocks to be digitized. These blocks range in size from fragments of less than $0,001 \mathrm{~m}^{3}$ to architectural blocks of more than one $\mathrm{m}^{3}$. Depending on the size and therefore the weight of the blocks, the work has been split into two groups: heavy blocks which cannot be moved and therefore remain at the repository and smaller blocks which can be easily carried to be digitized in the laboratory at INSA Strasbourg.

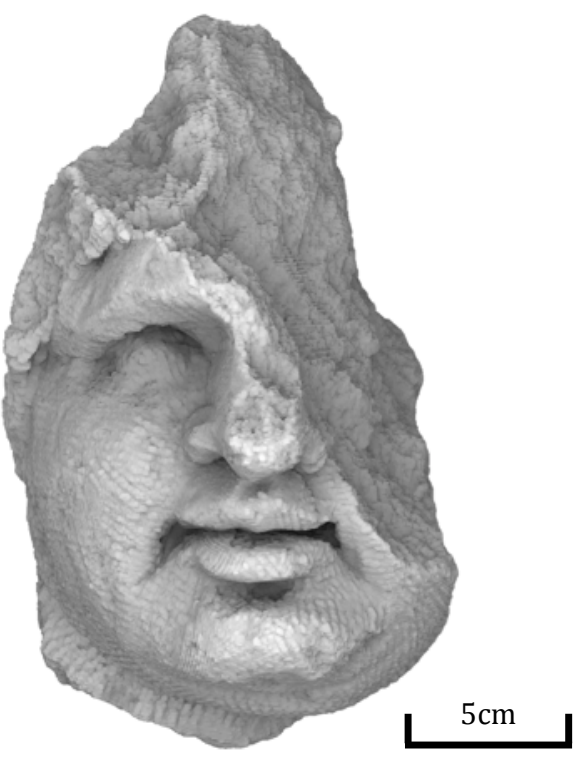

Figure 2. Head fragment, as an example of small object to record

The recording is thus made in two stages and at two different places. The small objects are digitized in the laboratory with a Scan Arm. For the recording of the largest blocks, two scientific missions have been planned at the repository near Paris to finalize the acquisition. Acquisition under laboratory conditions were spread over several weeks in contrast to the field operations carried out within a constrained period ( $2 \times 2$ days). In the lab, we used a FARO EDGE Scan Arm (accuracy of $0.034 \mathrm{~mm}$ ), in order to digitize the blocks with an accuracy of $0.1 \mathrm{~mm}$. The small blocks selected are often detailed, so it is important to sweep the entire surface. Depending on the complexity of the shape, two to three acquisitions in different block positions were required.

The recording missions at the repository were more stressing because of local constraints (space, light, etc.). We set up an effective survey, and close range photogrammetry has been applied. Indeed, this acquisition technique makes it possible to multiply the number of acquisition devices. Three cameras could be used simultaneously, namely a Canon EOS 5D Mark II camera with a sensor of 21 Megapixels and two Canon EOS 5D SR 50 cameras with a sensor of 50 Megapixels. A $28 \mathrm{~mm}$ fixed focal length lens was mounted on each SLR camera. In addition, two of the three devices were equipped with flash, in order to avoid the use of tripods and therefore speed-up the acquisition phase. The use of flash has an influence on the final result. This point will be discussed later. The large blocks have been affected by various deteriorations since they were stored in the repository. The limestone crumbled after drying. The blocks are breakable objects. They must be handled with care and cannot be recorded in different positions (such as the small blocks). Their position on the pallets at its initial storage was done in order to make visible the interesting parts. The hidden parts, which most often correspond to a contact surface with other blocks, are therefore not digitized. The number of shots depends on the shape of the object and the visible details. The number of photos can thus vary from simple to triple. The average number of images to be processed per block was about 140. The large mighty blocks are often split in several pieces. The fragments of these blocks have not been moved to the laboratory for the scan arm acquisition, that's why various acquisition projects with different configurations were needed. 
Experiments with a Faro Freestyle hand held scanner (Lachat, 2017) were carried out on the blocks but the data produced were only used for the assessment of the instrument. But this project shows again an example of combination of laser scanning (scan arm) and photogrammetry (Grussenmeyer et al., 2011) .

In the acquisition process, what is sought is the possibility of being able to use the 3D model for studies where the materiality of the real object is a constraint. Weight and size are the two main binding characteristics. Since the digital model must be as close as possible to the original, it must contain the actual dimensional characteristics and thus be scaled. The scaling of data from the two techniques used is not achieved in the same way. Laser scanning produces data whose dimensions correspond to reality. Therefore, no scaling is required. As described previously, several files corresponding to several positions of the object were produced. A registration is therefore necessary to obtain a single and coherent model for each object. In this case, the average error of aligning the point clouds was about $0.2 \mathrm{~mm}$.

For large blocks acquired by photogrammetry, scaling is a mandatory step in the post-processing chain applied to the original images. Distances measured in the model must be matched with known distances. This step was repeated for each block and has therefore been simplified. The protocol in place aims to automate the recognition of targets and the scaling of models. Rules of about 1 meter on which encoded targets are glued at calibrated distances were placed all around each block The use of encoded targets and their automatic recognition greatly contributes to the acceleration of the post-processing and to the achievement of an average scaling error of approximately $0.8 \mathrm{~mm}$.

The two types of data processed with the scan arm and the close range photogrammetry are in the form of point clouds. The scan arm produces position and intensity data while photogrammetry allowed the capture of colors of the objects. The anastylosis work is done on blocks resulting from the two acquisitions. The question of the homogeneous representation of the different elements was an important question in this project.

\section{DELIVERABLES AND RENDERING}

The representation of objects must be as efficient as possible. The visualization of the point clouds is fast and shows interesting aspects of the objects. The laser scanner directly produces this type of data and offers real-time visualization of results. Regarding photogrammetry, three-dimensional results are obtained only after the processing of the images.

To optimize the representation of the objects, the mesh rendering is close to the analogy with the surface of the object. It is calculated on the basis of the complete point cloud of the object. The representation of the mesh is made on the basis of the geometry or enriched by the texture of the images. The representation of the mesh is therefore either autonomous or dependent on the images and their characteristics.

Three types of images can be distinguished: outdoor with daylight, outdoor with flash or indoor with flash. The quality and contrast are therefore variable according to the intensity of the sunlight.

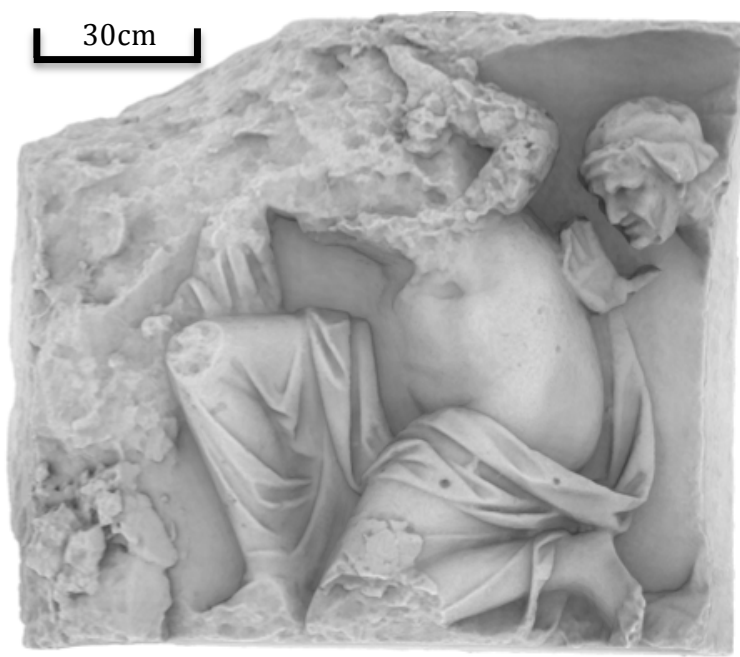

Figure 3. Mesh of a fragment of the frieze

The visual aspect of the models is also a very important issue. Indeed, textures from photos are an excellent way to bring about the realism of the virtual model, but fine details of the object are sometimes blurred by the uniformity of the color of the original material. Acquisition by hand-held scanner does not provide the textures (they must be acquired according to a complementary process). The data types are therefore different depending on the acquisition. The type of rendering of the models depends on precise choices to be defined optimally.

The texture of the object improves the realism but it is difficult to harmonize the radiometry, as some shadows present on the mesh are related to the lighting conditions during the acquisition. Either the contrast is high in areas with direct lighting or the texture is dark in the backlight. To obtain a harmonization of the representation of the meshes of the blocks, an artificial rendering is necessary.

Several methods of enhancing relief exist. Those based on geometrical characteristics, by normals simulating a projection of light analogous to sunlight, shading according to the point of view or optimizing the details. The normals are interesting as they are provided with the point clouds. The rendering is therefore interesting to quickly produce an overview of the mesh.

However, the details are not all highlighted in the same way. Shadows by directional light or by point of view are calculated in real time by the graphic card (Kilgard, 2000; Zhang et al., 2002). Like the previous one, they do not offer sufficient detail rendering. In order to generate a rendering that values the relief pertinently, the principle of ambient occlusion (Tarini et al., 2003) has been investigated.

The light comes from a sphere around the object, thus enhancing the relief of the object in the same way. Thanks to CloudCompare's proposed rendering mode (Duguet et al., 2004), the representation of objects by meshing makes the perception of the relief even more striking than the real object. It is certainly an artificial rendering, which does not benefit from the realism of the texture but allows to greatly enrich the perception of the relief, and brings another kind of fidelity to the representation of the blocks. 

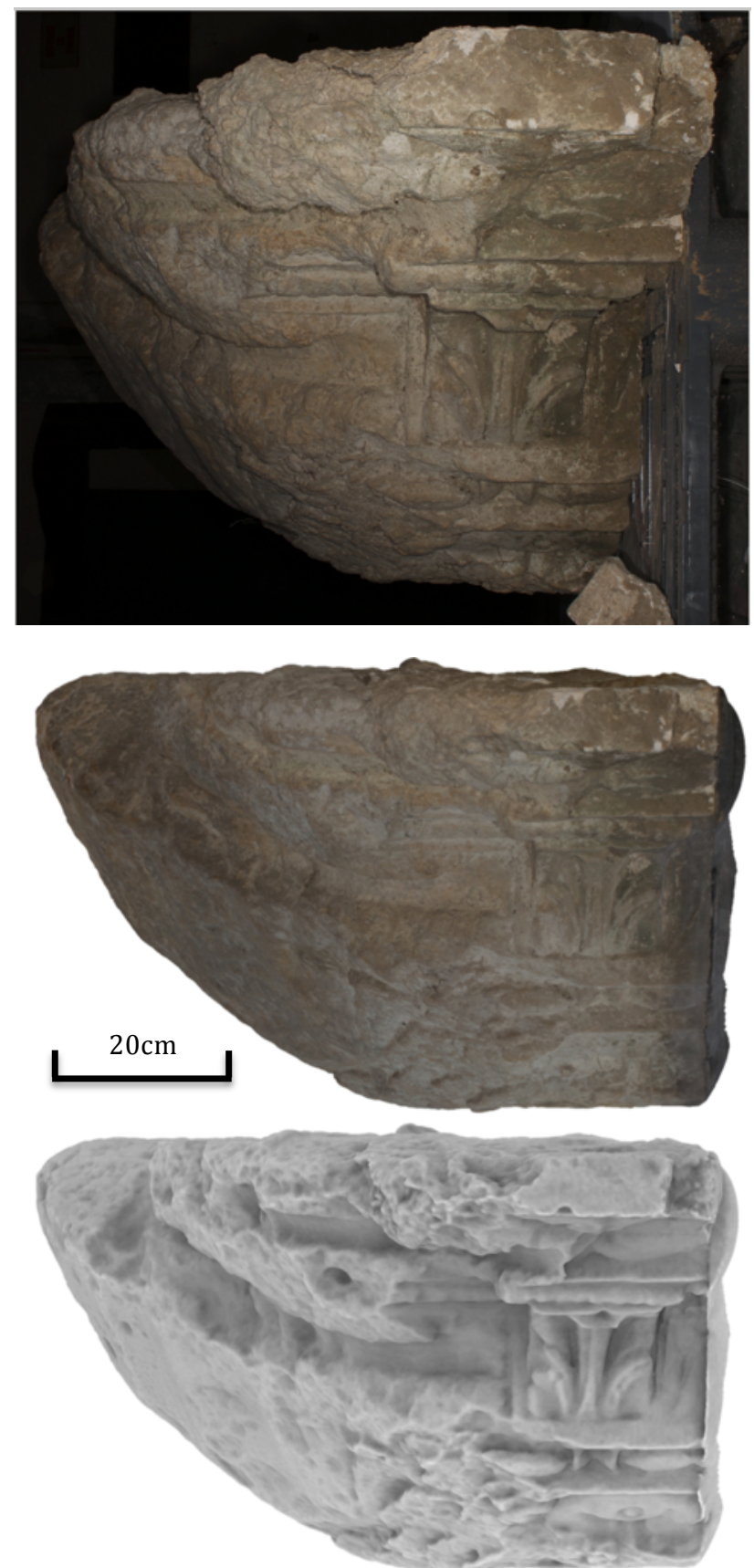

Figure 4. Various rendering results of a cornice block: From top to bottom: image from outdoor recording, texture 3D model, rendered model in PCV (portion of visible sky function in Cloud Compare)

\section{BLOCKS ANASTYLOSIS}

Some blocks broke after the fall of the facade. Most fragments were found nearby. If the size of the fragments allowed manipulation, anastylosis would have been done directly on the ground. As the number of fragments was large and the fragments shape complex, virtual anastylosis was required (unless to paste the pieces). The anastylosis of a block is interesting only for the remarkable blocks. Attention to a block is often related to its decor. Either the block is an architectural element that participates in the structure of the building, or it is a carved element of ornament. The architectural block has either a shape related to its structural function, or it is a carved ornamental element.
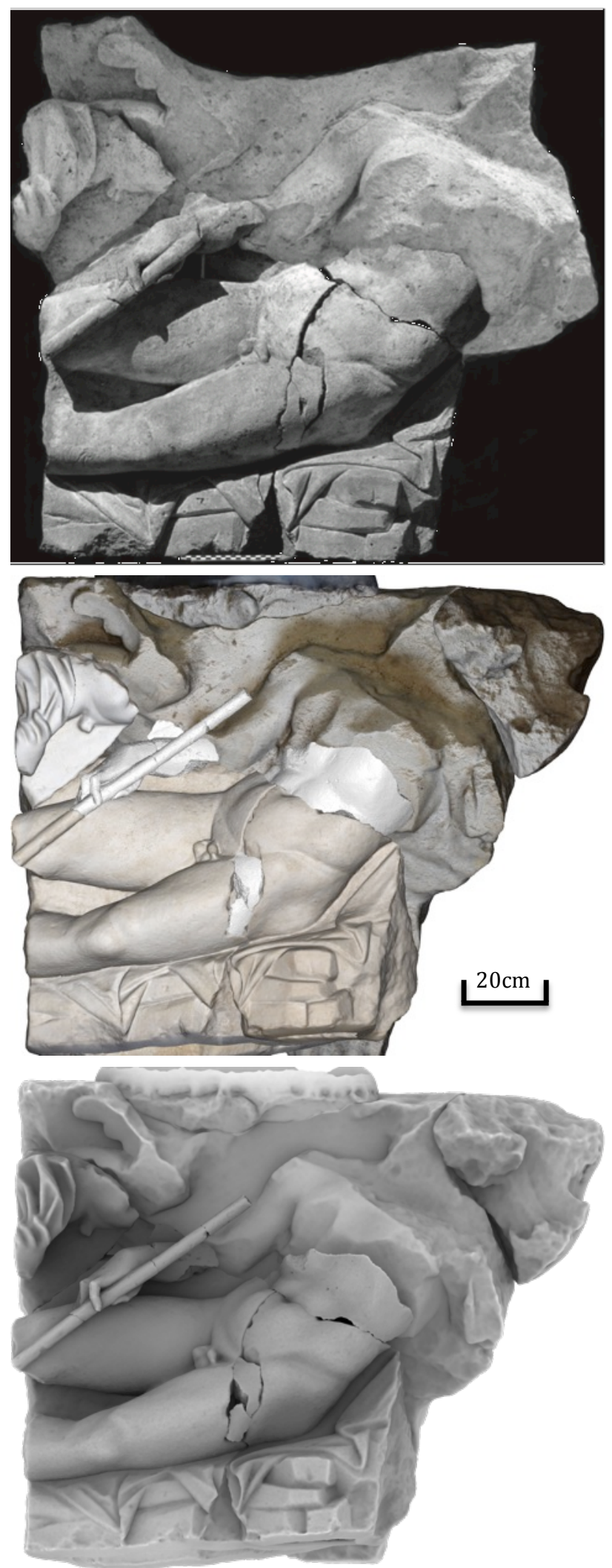

Figure 5. Top: reassembly of the blocks made on site (BrunetGaston, 2016)

In the middle: rendering of the virtual anastylosis of the block without color harmonization

Bottom: harmonization of anastylosis rendering by $\mathrm{PCV}$ 
The sculpted element represents a scene or symbolic elements. Its shape is imposed by these elements. The function of the block, whether structural or decorative, influences the overall shape of the block. It is necessary to take into account either its template, equal to a rectangular parallelepiped, or to an organic form that can, for example, be a monumental head or an imaginary animal. Structural blocks have higher resistance to ruin than figurative elements with less compact shapes. The quality of the limestone also affects the resistance of the blocks. A poor implementation and a variable quality of limestone could be noticed. Let us take the example of a characteristic feature of this facade: a griffin. The anastylosis of a griffin makes it possible to assemble a wing to its body. The connections between the wings and the body are very fragile without even considering the fall of the sculpture. In addition, the griffins at the top of the façade suffered the most devastating fall. None of them can be reconstituted in its entirety. But gathering as many fragments as possible makes it possible to better appreciate the quality of the sculptures.

Several types of anastylosis can be performed. They depend on the relative positioning of the fragments concerned. The fragments can be separated by a clear break. In a second case, the two fragments are juxtaposed, but the surface that binds them has been eroded. Finally the fragments belong to the same block but are not joined. These three physical relationships between fragments induce different modes of assembling.

The case of a sharp break is easy to manage with real fragments, regardless of their weight. Indeed, it is possible to find the ideal position, with effective means of validation. This perception of good assembling is not transposable numerically. The main criterion for an association of two fragments is the distance. The approach is related to the registration of two point clouds of the same object. It is nevertheless necessary to take the material into account. The surfaces of the fragments must be joined together. This is a different consideration from the registration where the error is averaged around the assumed position. In this case the objective is to minimize the positive distance of the two surfaces. In cases where the contact surfaces are eroded, the coincidence of the surfaces is important, but the continuity of the outer surface of the reconstituted block is critical. Since the fracture is more visible, the bias of the shape helps in the repositioning. The appreciation of the operator is therefore of great importance.

When the outer surface is a geometric shape (plane, cylinder), the assembling is simpler than an organic form.

\section{RECONSTRUCTION AND CONFRONTATION WITH DRAWINGS}

The shape of the blocks guides the anastylosis. There is always a potential error, but this relationship to material reduces significantly the probability of error. In order to propose hypothesis for the reconstruction of a façade of nearly 70 meters long by 10 meters high initially constituted by thousands of blocks, the uncertainty is much greater. The dimensions of the components and their relative positioning have to be taken into account. Knowledge of the regulating lines and stylistics of the period concerned are valuable guides. This knowledge is enhanced by the accumulation and the crosschecks of the archaeological discoveries. Variants are therefore possible.

The drawing of the complete elevation is carried out on the basis of the assumptions made in crosschecking the recovered elements. A reconstruction of the façade linked to the dimensional characteristics of the blocks and to their relative arrangement has been proposed. This drawing is based on measurements made by hand according to classical archaeological methods. The drawing can be carried out on projections made with respect to markers initially set up. The value of the design is more in the choice of the lines revealing the details of the blocks than in the precision of their position. The drawings are less accurate than usual archaeological documentation. The reconstruction of the facade is based on these drawings. The hypothetical dimensions of the building are thus derived from the accumulation of small dimensional differences with the materiality of the real object. Due to this accumulation, the difference with the stacking of the digitized blocks is significant. The drawing is the starting point of the three-dimensional reconstruction; it contains the knowledge of the archaeologist. The scanned blocks provide a dimensional reference to the built façade. Reconstruction should take into account the precise dimensions and archaeological information and propose a relevant synthesis of the two. The construction of the original façade is the result of the work of stonecutters. Their expertise is at the origin of the quality of the building. Nevertheless, the precision of alignment and respect of the dimensions throughout the construction of the structure is questionable. Although the digitization of the blocks reaches millimetric precision with regard to the tools used, the original construction cannot be so meticulous. In addition to measuring accuracy, consideration is given to the coherence between all the elements mentioned.

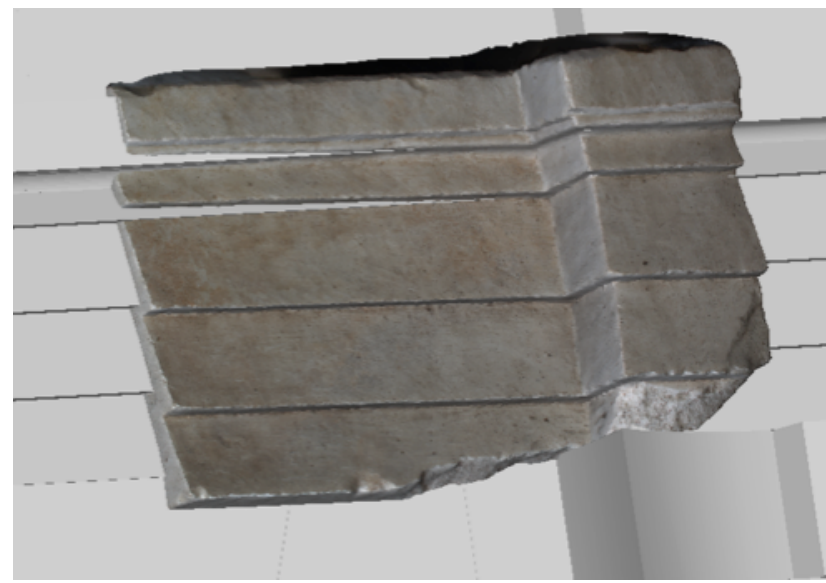

Figure 6. Portion of the reconstruction model made on the basis of the drawings with inlay of the digitized block, evidence of the offset $(\max 6 \mathrm{~cm})$

\section{OUTLOOK FOR THE MODEL AND CONCLUSION}

The blocks are derived from a fragile sedimentary rock; their numerous immersions due to the floods of the river located nearby have considerably weakened them. The drying during the storage in the repository takes place differently depending on the dimensions of the blocks and the differences in the degree of hygrometry. Several blocks are deteriorating slowly. Three-dimensional acquisition offers an opportunity to document these objects and to keep a representation of their state at a given time. Beyond this perpetuation, the project is not yet completed. Indeed, the exploitation of the model of the facade beyond a representation of a supposed state is in progress. The causes of the ruin of the facade are not yet certain. Several cases are possible but it is still difficult to decide between simulations and other possibilities to discover. A human demolition does not justify the desertion of the blocks in place, unless an unknown and very localized battle. A ruin by default of construction is not easily conceivable as the Roman works still visible today demonstrate the mastery of all stages of edification. A natural cause, combined with an exceptional 
climatic event associated with a soil constitution is evoked. The absence of information related to the ruin of the facade was an opportunity to use the digital model of the facade to try to get closer to a valid explanation. The model should thus be used to propose hypothesis explaining the fall of the blocks. Two approaches have been explored. The first is to generate a fall based on the model with assumption of effort. These efforts correspond to the causes of fall mentioned. It will then be necessary to compare the position of the blocks obtained once on the ground with the discovery positions of the blocks. The first method is related to the ability to apply fall models to the model. The second method proposes to use the two positions: the modeled one and the discovery façade, and to study the probable trajectories of a large number of blocks between the two. The combination of the two methods is also conceivable. Three-dimensional digitization is a mean of documenting the blocks difficult to preserve and affected by deteriorations in order to preserve the state at the time of the discovery. This project is primarily a resource to explain why this Gallo-Roman façade collapsed so shortly after its construction. This particular context gives us the opportunity to confront the 3D models to the drawings that were made of the blocks. $3 \mathrm{D}$ reconstruction is the product of the combination of knowledge integrated with the elevation of the proposed façade to the dimensional characteristics of the digitized blocks. This virtual façade will be used as a support for the elaboration of scenarios describing the ruin of the edifice.

\section{ACKNOWLEDGEMENTS}

The authors thank the EDF Corporate Foundation for financing this research. The discovery of the Pont-Sainte-Maxence site requires an exceptional restoration of blocks, a better understanding of the organization and the evolution of the site and further pigment studies. The EDF Corporate Foundation, by financing these studies, makes the best possible use of these remains.

\section{BIBLIOGRAPHY}

Brunet-Gaston V, Gaston C., JOBIC F., et alii. « Les dieux du stage de Sarron: une façade monumentale gallo-romaine d'exception » Rapport de fouille, Pont-Sainte-Maxence, NordPicardie « Hauts-de-France, Oise, Inrap 2016. 3 volumes.

Duguet, F., Girardeau-Montaut, D., Rendu en Portion de Ciel Visible de Gros Nuages de Points 3D. In Actes des journées de l'AFIG, Nov 2004, Poitiers, France. pp.11, 2004.

Grussenmeyer, P., Alby, E., Assali, P., Poitevin, V., Hullo,J.-F., Smigiel, E., 2011. Accurate documentation in cultural heritage by merging TLS and high-resolution photogrammetric data. SPIE Optical Metrology 2011, Munich 23-26 May, Videometrics, Range Imaging, and Applications XI, Proc. of SPIE Vol. 8085, 808508 - C 2011 SPIE - CCC code: 0277786X/11/\$18 doi: 10.1117/12.890087, 13 pages.

Kilgard, M. J. 2000. A Pratical and robust bump mapping technique for today's GPU's. In: Game Developers Conference. Advanced Opengl Game development (July 2000). Online: http://www.nvidia.com

Lachat. E., Landes, T., Grussenmeyer, P., 2017. Performance investigation of a handheld $3 \mathrm{D}$ scanner to define good practices for small artefact 3D modeling. In International Archives of the Photogrammetry, Remote Sensing and Spatial Information Sciences, $26^{\text {th }}$ CIPA Symposium, Ottawa, Canada.
Tarini, M., Cignoni, P., Scopigno, R. , 2003.Visibility based methods and assessment for detail-recovery. In Proc. Of Visualization, Seattle, USA, (ISBN 0-7695-2030-8), 457-464.

Zhang, E., Turk, G. 2002. Visibility-guided simplification. In Proc. of the 13th IEEE Visualization Conf. (VIS-02), IEEE Computer Society, Piscataway, NJ, R. Moorhead, M. Gross, and K. I. Joy, Eds., 267-274. 